\title{
VALOR PROGNÓSTICO DA ESPESSURA TUMORAL NO CARCINOMA EPIDERMÓIDE DE BOCA E OROFARINGE
}

\section{PROGNOSTIC VALUE OF TUMOR THICKNESS IN SQUAMOUS CELL CARCINOMA OF THE MOUTH AND OROPHARYNX}

\author{
Ali Amar ${ }^{1}$ \\ Abrão Rapoport, TCBC ${ }^{2}$ \\ Marcos Brasilino de Carvalho ${ }^{3}$
}

\begin{abstract}
RESUMO: Objetivo: Avaliar o significado prognóstico da espessura tumoral no carcinoma epidermóide de boca e orofaringe. Método: Foram avaliados retrospectivamente 112 pacientes atendidos entre janeiro de 1990 e dezembro de 1994, dos quais foram selecionados 84 casos com amostras teciduais disponíveis. A medida da espessura foi realizada à microscopia óptica com ocular milimetrada, considerando a maior medida perpendicular à superfície. Resultados: A incidência de metástases ocultas foi de 7\% nos tumores menos espessos do que $3 \mathrm{~mm}$ e $50 \%$ naqueles com espessura igual ou superior a $5 \mathrm{~mm}$. As margens cirúrgicas estiveram comprometidas com maior freqüência nos pacientes com espessura tumoral maior ou igual a $5 \mathrm{~mm}$, mas a espessura não identificou os pacientes com maior risco de recidiva local. Conclusão: A espessura tumoral auxilia a identificar os pacientes com carcinoma epidermóide de boca e orofaringe com maior risco de metastatização oculta.
\end{abstract}

Descritores: Prognóstico; Espessura; Carcinoma epidermóide.

\section{INTRODUÇÃO}

Há uma busca incessante de fatores prognósticos que possam contribuir para o planejamento terapêutico de pacientes com câncer, identificando aqueles com maior probabilidade de se beneficiarem com as diferentes opções de tratamento. Embora o estadiamento TNM persista como o indicador prognóstico mais significativo, a espessura ou volume tumoral podem fornecer informações adicionais a respeito da doença. A espessura tumoral se revelou um indicador prognóstico em outros sítios anatômicos, como pele e colo uterino, e também mostrou relação com a metastatização nos tumores iniciais de língua e soalho ${ }^{1,2}$.
Este estudo tem por objetivo avaliar o significado prognóstico da espessura tumoral no carcinoma epidermóide do andar inferior da boca e orofaringe.

\section{MÉTODO}

Foram avaliados retrospectivamente 112 casos de carcinoma epidermóide do andar inferior da boca e da orofaringe tratados no Serviço de Cirurgia de Cabeça e Pescoço do Hospital Heliópolis no período de janeiro de 1990 a dezembro de 1994.

A análise histológica empregou cortes teciduais corados pela hematoxilina-eosina, avaliados à microscopia óptica. A espessura do tumor considerou a maior medida

1. Cirurgião e Mestre em Cirurgia de Cabeça e Pescoço, Hospital Heliópolis, São Paulo, SP.

2. Coordenador do Curso de Pós-Graduação, Hospital Heliópolis, São Paulo, SP.

3. Chefe do Serviço de Cirurgia de Cabeça e Pescoço, Hospital Heliópolis, São Paulo, SP.

Recebido em 09/01/2001

Aceito para publicação em 18/10/2001

Trabalho realizado no Serviço de Cirurgia de Cabeça e Pescoço do Complexo Hospitalar Heliópolis, Hosphel, São Paulo. 
a partir da superfície ulcerada, empregando uma ocular milimetrada. A medida da espessura somente pôde ser realizada em 84 casos, dos quais foram recuperadas as amostras teciduais.

Foi considerado o estadiamento clínico e patológico conforme os critérios da classificação TNM da UICC/ AJCC - 1997.

A espessura tumoral foi classificada em três categorias: $<3 \mathrm{~mm}, 3$ a $4,9 \mathrm{~mm}, 5 \mathrm{~mm}$ ou mais. As categorias foram relacionadas com a presença de metástases linfáticas, comprometimento de margens cirúrgicas e recidiva local.

Entre os 84 pacientes, 75 eram do sexo masculino e nove do sexo feminino. A idade média foi de 55 anos. $\mathrm{O}$ sítio primário predominante foi a língua e soalho com 40 casos, 21 casos com lesão em gengiva e área retromolar e 23 casos de lesões da orofaringe. O tempo de seguimento apresentou média de 33 meses (1-113).

A análise estatística empregou o teste de Qui quadrado e o teste exato de Fisher, considerando significativas as diferenças com erro alfa inferior a $5 \%$.

\section{RESULTADOS}

Houve maior percentual de casos $\mathrm{pN}+$ nas lesões com espessura $\geq 5 \mathrm{~mm}$, mas $43 \%$ dos tumores com espessura inferior a $3 \mathrm{~mm}$ também apresentaram metástases linfonodais (Tabela 1).
Nos casos N0 houve apenas 7\% de metástases ocultas em pacientes com tumores menos espessos do que $3 \mathrm{~mm}$ e $50 \%$ naqueles com espessura igual ou superior a $5 \mathrm{~mm}$. Nos casos $\mathrm{N}+$ a espessura tumoral não auxiliou a identificar os casos pNo (falso-positivos). (Tabela 2).

A incidência de margens cirúrgicas comprometidas (Tabela 3) foi de 3\% nos tumores com espessura inferior a $5 \mathrm{~mm}$ e $20 \%$ naqueles com espessura igual ou maior a $5 \mathrm{~mm}$.

A espessura mostrou relação com a recidiva local apenas nas lesões de boca, porém estatisticamente não significativa $(\mathrm{p}=0,44)$, conforme Tabela 4 tanto para a boca quanto para a orofaringe.

\section{DISCUSSÃO}

A avaliação da espessura tumoral necessita de cortes seriados do tumor primário para que seja avaliada de forma fidedigna. Obviamente a diferença entre seu componente infiltrativo e vegetante também deve ser considerada, mas é difícil de ser avaliada em estudos retrospectivos realizados com pequena amostra tecidual. A capacidade de o tumor produzir seu próprio estroma ou sua interação com o tecido conjuntivo adjacente pode estar relacionada com uma diferença funcional entre as lesões infiltrativas ou vegetantes.

A drenagem linfática ocorre a partir da submucosa, de forma que mesmo lesões pouco espessas atingem rapi-

Tabela 1

Espessura tumoral e metastatização

\begin{tabular}{l|l|l|l|l}
\hline & $p N O$ & $p N+$ & $R R$ & \multicolumn{1}{c}{$p$} \\
\hline$<3 \mathrm{~mm}$ & 18 & 14 & ref. & - \\
$3-4,9 \mathrm{~mm}$ & 16 & 14 & $1,05(0,67-1,66)$ & 0,98 \\
$\geq 5 \mathrm{~mm}$ & 9 & 16 & $1,56(0,85-2,86)$ & 0,12 \\
\hline
\end{tabular}

Tabela 2

Espessura tumoral e metastatização em pacientes NO

\begin{tabular}{l|l|l|l|l|l}
\hline & $N O$ & $p N O$ & $p N+$ & $R R$ & $p$ \\
\hline$<3 \mathrm{~mm}$ & 14 & 13 & 1 & ref. & - \\
$3-4,9 \mathrm{~mm}$ & 13 & 10 & 3 & $1,21(0,87-1,68)$ & 0,24 \\
$\geq 5 \mathrm{~mm}$ & 10 & 5 & 5 & $1,86(0,98-3,51)$ & 0,02 \\
\hline
\end{tabular}

Tabela 3

Espessura tumoral e margens cirúrgicas

\begin{tabular}{l|cc|cc|cc}
\hline & \multicolumn{2}{|c|}{ Margem (+) } & \multicolumn{2}{c}{ Margem(-) } & RR & $p$ \\
\hline$<3 \mathrm{~mm}$ & 1 & $(3 \%)$ & 31 & $(97 \%)$ & ref. & - \\
$3-4,9 \mathrm{~mm}$ & 1 & $(3 \%)$ & 29 & $(97 \%)$ & $1,07 \quad(0,07-16,30)$ & 1,00 \\
$\geq 5 \mathrm{~mm}$ & 5 & $(20 \%)$ & 20 & $(80 \%)$ & $6,20(1,29-29,88)$ & 0,01 \\
\hline
\end{tabular}


Tabela 4

Espessura tumoral e recidiva local

\begin{tabular}{|c|c|c|c|c|}
\hline & \multicolumn{4}{|c|}{ Recidiva Local } \\
\hline & & Sim & & Não \\
\hline \multicolumn{5}{|l|}{ Boca* $^{*}$} \\
\hline$<3 \mathrm{~mm}$ & 7 & $(29 \%)$ & 17 & $(71 \%)$ \\
\hline $3-4,9 \mathrm{~mm}$ & 7 & $(33 \%)$ & 14 & $(67 \%)$ \\
\hline$\geq 5 \mathrm{~mm}$ & 9 & $(47 \%)$ & 10 & $(53 \%)$ \\
\hline \multicolumn{5}{|c|}{ Orofaringe } \\
\hline$<3 \mathrm{~mm}$ & 4 & $(50 \%)$ & 4 & $(50 \%)$ \\
\hline $3-4,9 \mathrm{~mm}$ & 3 & $(33 \%)$ & 6 & $(67 \%)$ \\
\hline$\geq 5 \mathrm{~mm}$ & 1 & $(16 \%)$ & 5 & $(84 \%)$ \\
\hline
\end{tabular}

$* p=0,44$

damente as vias linfáticas. Os tumores menos espessos apresentam menor potencial de metastatização e de recidiva local, mas o fato de $43 \%$ dos tumores com espessura inferior a $3 \mathrm{~mm}$ apresentarem metástases sugere que a metastatização seja um evento precoce nos carcinomas epidermóides das vias aerodigestivas superiores.

Um maior componente infiltrativo é uma característica das lesões de estadiamento T4. Este comportamento reflete a produção de integrinas e metaloproteinases que permite ao tumor infiltrar e proliferar no tecido adjacente, uma qualidade necessária aos clones metastáticos ${ }^{3,4}$. Esta característica tumoral pode ser mais significativa do que o crescimento longitudinal. Este último parâmetro não reflete necessariamente a maior proliferação, uma vez que pode haver a coalescência de focos multicêntricos ${ }^{5}$. Além disto, a metastatização constitui o principal fator prognóstico nos carcinomas, responsável pela baixa eficiência da terapia localizada.

Diferentes autores empregaram diversos pontos de corte na tentativa de definir uma espessura associada com bom ou mau prognóstico, variando desde 1,5 até $10 \mathrm{~mm}^{6-}$ 11. O ponto de corte é arbitrário, temos a definição de extremos que se correlacionam com o risco de metastatiza- ção e prognóstico, mas os valores intermediários podem ser de pouca utilidade na definição do prognóstico individual, representando um artefato estatístico ${ }^{12}$. Os estudos realizados até o momento não apresentaram casuística com tamanho adequado para esta estimativa. A inclusão de diversos subsítios também dificulta a interpretação dos resultados, uma vez que a mucosa pode apresentar espessura diferente. Como a maior utilidade reside na identificação do risco de metástases ocultas, a espessura deve ser avaliada no pré-operatório. A ultra-sonografia pode ser empregada para este fim, mas não apresenta vantagens sobre a palpação da lesão realizada sob narcose, pois uma espessura intermediária não tem um significado claro ${ }^{13}$. Além de uma estimativa da espessura, a palpação pode definir a consistência do tumor, um parâmetro que também pode ajudar a prever o risco de metastatização oculta, relacionado com a proporção entre células e estroma ${ }^{14}$. Esta avaliação tem aplicação apenas em tumores no estádio T1 e T2, já que não é indicada a conduta expectante no pescoço em lesões primárias maiores. Entre os pacientes com CEC de boca que persistem com o pescoço clinicamente negativo a despeito de uma lesão primária maior, é observado percentual semelhante de falso-negativos entre aqueles com tumor nos estádios T2, T3 ou T4 (dados não publicados do Serviço de Cirurgia de Cabeça e Pescoço do Hospital Heliópolis).

No presente estudo todos os pacientes foram submetidos a esvaziamento cervical, com diagnóstico de metástases ocultas em $24 \%$ dos casos, mas o significado clínico das eventuais micrometástases ainda não foi elucidado. A espessura pode ajudar a definir os casos T1 que merecem um esvaziamento eletivo e os casos T2 que poderão ser preservados, porém a baixa morbidade de um esvaziamento seletivo permite ampliar a sua indicação.

Não foi possível identificar uma correlação entre a espessura tumoral e a recidiva local (Tabela 4). Apesar do maior percentual de comprometimento da margem cirúrgica nos tumores com espessura maior ou igual a $5 \mathrm{~mm}$ (Tabela 3), este fator não é um indicador fidedigno do risco de recidiva ${ }^{15}$. Além disto, muitos pacientes nesta casuística foram perdidos de seguimento precocemente, impedindo uma análise deste parâmetro.

\begin{abstract}
Background: To evaluate the prognostic meaning of tumor thickness in squamous cell carcinoma of the mouth and oropharynx. Method: A retrospective study of 112 patients treated between January 1990 and December 1994 was undertaken and 84 cases with tecidual samples were selected. Tumor thickness was measured by optical microscopy, considering the largest perpendicular measure. Results: The incidence of occult metastases was $7 \%$ in tumors less than $3 \mathrm{~mm}$ and $50 \%$ in those $5 \mathrm{~mm}$ or more in thickness. Surgical margins were compromissed more frequently in patients with tumor thickness of $5 \mathrm{~mm}$ or more, but mesure of tumor thickness was not prone to identify patients with larger risk of local recurrence. Conclusion: Tumor thickness aids to identify patients with squamous cell carcinoma of the mouth and oropharynx with higher metastatic risk.
\end{abstract}

Key Words: Prognostic; Thickness; Squamous cell carcinoma 


\section{REFERÊNCIAS}

1. Breslow A. Thickness, cross-sectional area and depth of invasion in the prognosis of cutaneous melanoma. Ann Surg 1970; 172: 902-8

2. Asakage T, Yokose T, Mukai K, et al. Tumor thickness predicts cervical metastasis in patients with stage I/II carcinoma of the tongue. Cancer 1998; 82:1443-8.

3. Kurahara S, Shinohara M, Ikebe T. Expression of MMPs, MT-MMP, and TIMPs in squamous cell carcinoma of the oral cavity: correlations with tumor invasion and metastasis. Head Neck 1999; 21: 627-38.

4. Mizejewski G. Role of integrins in cancer: survey of expression patterns. Proc Soc Exp Biol Med 1999;222:124138.

5. Slaughter DP, Southwick HW, Smejkal W. Field cancerization in oral stratified squamous epithelium. Cancer 1953; 6:963-8

6. Morton RP, Ferguson CM, Lambie NK, et al. Tumor thickness in early tongue cancer. Arch Otolaryngol 1994;120:717-20.

7. Fakih AR, Rao RS, Borges AM, et al. Elective versus therapeutic neck dissection in early carcinoma of the tongue. Am J Surg 1989; 158:309-13.

8. Spiro RH, Huvos AG, Wong GY, et al. Predictive value of tumor thickness in saquamous carcinoma confined to the tongue and floor of the mouth. Am J Surg 1986; 152:345-50.

9. Mohit-Tabatabai MA, Sobel HJ, Rush BF, et al. Relation of thickness of floor of mouth stage I and II cancers to regional metastases. Am J Surg 1986; 152:351-3.

10. Moore C, Kuhns JG, Greenberg RA. Thickness as prognostic aid in upper aerodigestive tract cancer. Arch Surg 1986; 121:1410-4.

11. Yamasaki H, Inoue T, Teshima T. Tongue cancer treated with brachytherapy: is thickness of tongue cancer a prognostic factor for regional control? Anticancer Res 1998;18:1261-5.
12. Altman DG, Lausen B, Sauerbrei W. Dangers of using "optimal" cutpoints in the evaluation of prognostic factors. J Natl Cancer Inst 1994; 86:829-35.

13. Ogura I, Amagasa T, Miyakura T. Correlation between tumor consistency and cervical metastasis in tongue carcinoma. Head Neck 2000;22:229-33.

14. Shintani S, Nakayama B, Matsuura $H$, et al. Intraoral ultrasonography is useful to e valuate tumor thickness in tongue carcinoma. Am J Surg1997; 173:345-7.

15. Jones AS, Bin Hanafi Z, Nadapalan V. Do positive ressection margins after ablative surgery for head and neck cancer adversely affect prognosis? A study of 352 patients with recurrent carcinoma following radiotherapy treated by salvage surgery. British J Cancer 1996; 128-32.

Endereço para correspondência:

Prof. Dr. Abrão Rapoport

Praça Amadeu Amaral, 47 - cj. 82

01327-010 — São Paulo-SP

E-mail: cpgcp.hosphel@attglobal.net 